* Corresponding author E-mail address: gabriela.izarikova@tuke.sk (G. Ižaríková)

Article information Article history: AMS-Volume15-No.2-00109-11 Received 12 April 2011 Accepted 12 May 2011 Available online 26 August

\section{The Model of Topological Relations-Relation Between Two Constructed Equipment}

\author{
Kováč Jozef ${ }^{b}$, Knežo Dušan ${ }^{\text {a }}$, Ižaríková Gabriela ${ }^{\text {a* }}$
}

a Department of Applied Mathematics and Informatics, Faculty of Mechanical Engineering, Letná 9, 04187 Košice, Slovak Republic

${ }^{b}$ Department of Industrial Engineering and Management, Faculty of Mechanical Engineering, Nemcovej 32, 04200 Košice, Slovak Republic

\section{BIOGRAPHICAL NOTES}

Jozef Kováč, prof. Ing. CSc. is a professor of the automation and control. He is responsible for the study program Industrial engineering. He is head of Department of Management and Economics, Faculty of Mechanical Engineering, Technical University in Košice. He deals with the science activities in industrial engineering, innovation and automation of production and engineering activities. He is focusing on assembly and disassembly systems and the utilization of the virtual reality in the planning of production systems.

Dušan Knežo, prof. RNDr. CSc. (born 1956) graduated at the Faculty of Science in Safarik University in Košice in 1980. He defended his RNDr. at the Šafárik University in Košice in the year 1982. He defended PhD. in the field specializated in the Mathematical analysis in 1994. In 1998 he made the habilitation at the University of Žilina in the field specialized of the Applied mathematics. He was appointed Professor in the field of study "Biomedical engineering" in the year 2011. Since 1980 he is working in the Department of Applied Mathematics, Faculty of Mechanical Engineering as assistant, professional assistant and associate professor. He was a vice-dean for pedagogy at the Faculty of Mechanical Engineering, Technical University in Košice and at this time he works as a head of Department of Applied Mathematics and Informatics. Prof. Knežo published more than 60 publications in Slovak and international journals.

Gabriela Ižaríková, Mgr. PhD. (born 1974) graduated at the Faculty of Science in Safarik University in Košice (teacher of mathematics and physics) in 1997. She has worked at Department of Applied Mathematics at the Faculty of Mechanical Engineering, Technical University in Košice since 1997. She received PhD. in 2009 at the Faculty of Mechanical Engineering, Technical University in Kosice. Nowadays she works as a lecturer in the above mentioned department. Her research interests are focused on addressing the relationships inside the zone topology problems.

\section{KEY WORDS}

Assembled System, Constructed Equipments, Model of Topological Relations

\section{ABSTRACT}

Application of topological models for automated planning of sub-assembled systems is a new trend in design process. The paper deals with mathematical models of topological relations between distributed constructed equipments of assembled systems.

\section{Introduction}

At the present time of the development of scientific-technical revolution necessity 
of new access in all stages of the production is entering forward. The automatization introduces major means for increasing of the productivity, quality and competitive capacity of the production and services. Significantly increase productivity of work, increase quality, save resources, energy and life environment is possible only with well-designed, well-structured and optimized production systems.

A variety of activities and problems, connected with designing of assembled systems, are necessary to solve in conceptual and particular phase of solution.

The solution of zonal problems and dislocating task is at work out model expressive technological-organizational essence of assembled process by structure, which is displayed by layout and time arrangement of constructed equipments.

The study confirmed that considerable attention of this problem will be devoted to solution of topological relations within zonal problems.

\section{The Methodical Progresses of Automated Designing}

The methodical progresses of automated designing are based on the utilizing of topological models of constructed equipments of assembled systems. These progresses go out these assumptions:

- The model of raster grid is assigned to reference area for location assembled system. This system is laid out capacitive calculation of required surface (volume).

- Reference area of assembled system is located to the reference area by the assembled activity.

- The models of topological relations of the constructed equipments of assembled models are solved in the individual zones.

- The calculation-interactive mode of the solution of topological relations is based on the utilizing $2 D$ or $3 D$ models of constructed equipments of topological relations.

- The concrete $2 D$ and $3 D$ models are chosen from graphic database and inserted into calculated reference points.

- The optimal variant of structure of the assembled model is chosen on the base of interactive decision progress.

\section{The Model of Topological Relations-Relation Between Two Constructed Equipment}

The base of solution is model of topological system of two constructed equipments of structure assembled model. On the basis of combinatory principle is possible to create variously variants of complex structures of assembled systems from based model. The solution is defined by further stages:

A. The all parts of located constructed equipments are approximated to the cuboids with cants parallel with axes $x_{\boldsymbol{r}}$

$\mathbf{y}, \mathbf{z}$. The parameters and location of everyone cuboids is given by the coordinates of its cants. All three-dimensional constructed equipments and objects in the plain are approximated by the rectangle. Syntax for the determination parameters of assembled system:

$\mathrm{x}_{\mathrm{i}+}$ - dimension in positive course of $\mathrm{x}$-axe,

$\mathrm{x}_{\mathrm{i}-\mathrm{i}}$ - dimension in negative course of $\mathrm{x}$-axe,

$\mathrm{y}_{\mathrm{i}+}$ - dimension in positive course of $\mathrm{y}$-axe,

$\mathrm{y}_{\mathrm{i}-\mathrm{i}}$ - dimension in negative course of $\mathrm{y}$-axe,

$\mathrm{X}_{\mathrm{iB}} \mathrm{y}_{\mathrm{iB}}$ - parameters of safety zone,

$\mathrm{x}_{\mathrm{i}^{\prime}} \mathrm{y}_{\mathrm{i}}$ - coordinates of charging point,

$\mathrm{x}_{\mathrm{ij}}$ - distance between $\mathrm{i}$ and $\mathrm{j}$ object in course of

$\mathrm{x}$-axe,

$\mathrm{y}_{\mathrm{ij}}$ - distance between $\mathrm{i}$ and $\mathrm{j}$ object in course of y-axe,

$\mathrm{i}, \mathrm{j}$ - ordered number,

$\mathrm{d}$ - distance between charging points,

$\alpha$ - angle.

The charging point of constructed equipment of assembled system is located on the axe $o_{x^{*}}$ (Fig. 1)

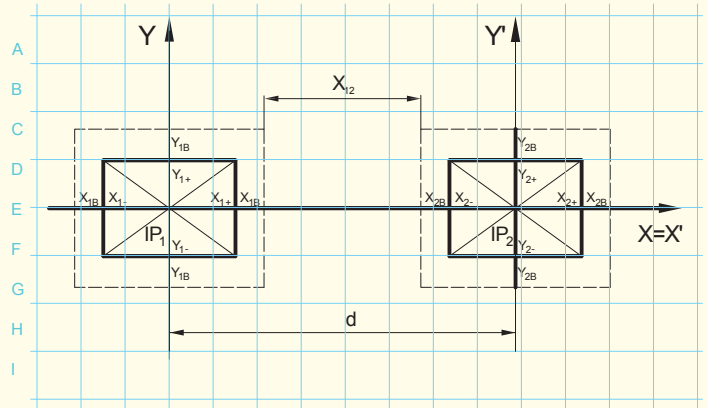

Fig. 1: Model of topological relations $1-O_{x}$

$$
\begin{aligned}
& I P_{1}=\left[x_{1}, y_{1}, 0\right]=[0,0,0] \quad I P_{2}=\left[x_{2}, y_{2}, 0\right] \\
& \alpha=0 \\
& x_{2}=x_{1+}+x_{1 B}+x_{2 B}+x_{2-}+x_{12} \quad y_{2}=y_{1}=0 \\
& x_{12}=d-\left(x_{1+}+x_{1 B}+x_{2 B}+x_{2-}\right) \\
& x_{12}=d-\left(x_{1+}+x_{1 B}+x_{2 B}+x_{2-}\right)
\end{aligned}
$$

$\mathrm{x}_{12}>0$ - this condition must be done because of the collision of constructed equipments of assembled system. 
The charging point of constructed equipment of assembled system is located on the axe $o_{y}$ : (Fig. 2)

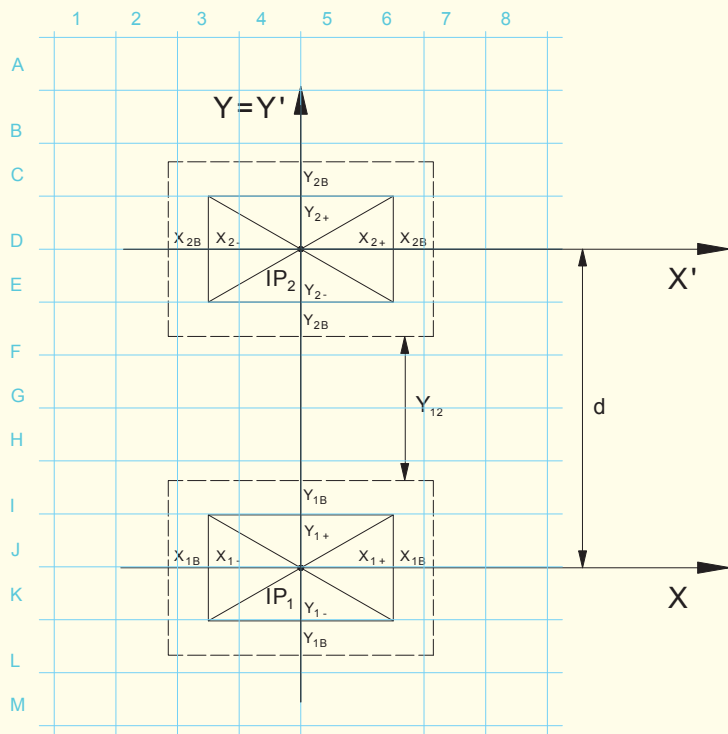

Fig. 2: Model of topological relations $1-O_{y}$

$$
\begin{aligned}
& I P_{1}=\left[x_{1}, y_{1}, 0\right]=[0,0,0] \quad I P_{2}=\left[x_{2}, y_{2}, 0\right] \\
& \alpha=\frac{\pi}{2} \\
& x_{2}=x_{1}=0 \quad y_{2}=y_{1+}+y_{1 B}+y_{2 B}+y_{2-}+y_{12} \\
& d=y_{1+}+y_{1 B}+y_{2 B}+y_{2-}+y_{12} \\
& y_{12}=d-\left(y_{1+}+y_{1 B}+y_{2 B}+y_{2-}\right)
\end{aligned}
$$

$\mathrm{y}_{12}>0$ - this condition must be done because of the collision of constructed equipments of assembled system. - The charging point of constructed equipment of assembled system turned about angle $\alpha, \alpha$-angle of join charging points and axe $o_{x}$ : (Fig. 3)

$$
\begin{aligned}
& I P_{1}=\left[x_{1}, y_{1}, 0\right]=[0,0,0] \quad I P_{2}=\left[x_{2}, y_{2}, 0\right] \\
& \alpha \in\left(0, \frac{\pi}{2}\right) \quad \alpha=\operatorname{arctg} \frac{y_{2}}{x_{2}} \\
& x_{2}=x_{1+}+x_{1 B}+x_{2 B}+x_{2-}+x_{12} \\
& y_{2}=y_{1+}+y_{1 B}+y_{2 B}+y_{2-}+y_{12} \\
& d=\sqrt{x_{2}^{2}+y_{2}^{2}} \\
& =\left(\left(x_{1+}+x_{1 B}+x_{12}+x_{2 B}+x_{2-}\right)^{2}\right. \\
& \left.\quad+\left(y_{1+}+y_{1 B}+y_{12}+y_{2 B}+y_{2-}\right)^{2}\right)^{\frac{1}{2}} \\
& x_{12}=x_{2}-\left(x_{1}+x_{1 B}+x_{2 B}+x_{2-}\right) \\
& y_{12}=y_{2}-\left(y_{1+}+y_{1 B}+y_{2 B}+y_{2-}\right)
\end{aligned}
$$

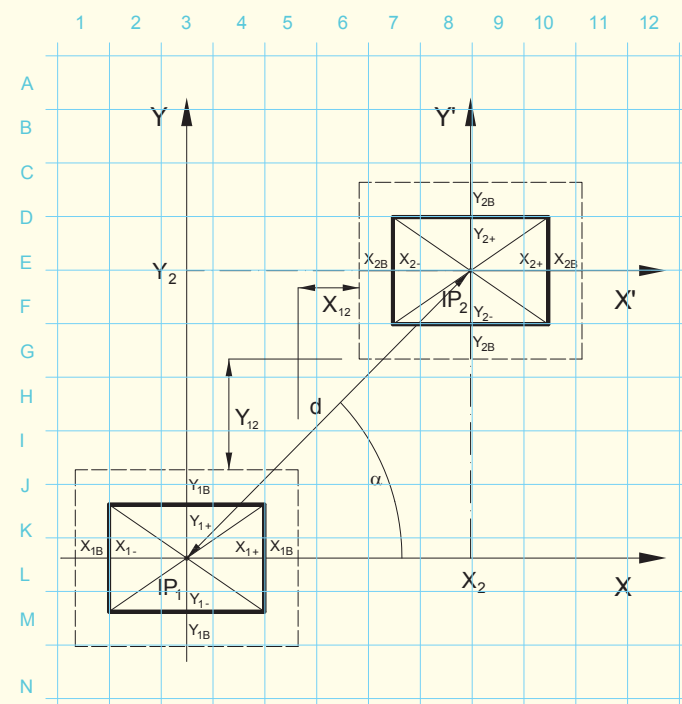

Fig. 3: Model of topological relations $1-\alpha$

$\mathrm{x}_{12} \geq 0 \vee \mathrm{y}_{12} \geq 0\left(\mathrm{x}_{12} \neq 0 \wedge \mathrm{y}_{12} \neq 0\right)$ - these conditions must be done because of the collision of constructed equipments of assembled system.

B. The all parts of located constructed equipments are approximated to the sphere with radius $r_{i}, i=1,2, \ldots$, and are approximated in the plain to the disk, and its cover curve is formed by circle.

$$
\begin{aligned}
& r_{1}=x_{1+}=x_{1-}=y_{1+}=y_{1-} \\
& r_{2}=x_{2+}=x_{2-}=y_{2+}=y_{2-} \\
& x_{1 B}=y_{1 B} \\
& x_{2 B}=y_{2 B}
\end{aligned}
$$

The charging point of constructed equipment of assembled system is located on the axe $o_{x}$ : (Fig. 4)

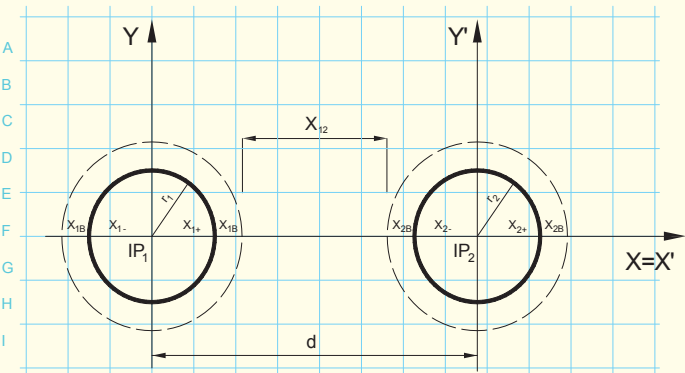

Fig. 4: Model of topological relations $2-O_{x}$ 


$$
\begin{aligned}
& I P_{1}=\left[x_{1}, y_{1}, 0\right]=[0,0,0] \quad I P_{2}=\left[x_{2}, y_{2}, 0\right] \\
& \alpha=0 \\
& x_{2}=r_{1}+x_{1 B}+x_{2 B}+r_{2}+x_{12} \quad y_{2}=y_{1}=0 \\
& d=r_{1}+x_{1 B}+x_{2 B}+r_{2}+x_{12} \\
& x_{12}=d-\left(r_{1}+x_{1 B}+x_{2 B}+x_{2}\right)
\end{aligned}
$$

$\mathrm{x}_{12}>0$ - this condition must be done because of the collision of constructed equipments of assembled system.

- The charging point of constructed equipment of assembled system is located on the axe $o_{y}:$ (Fig. 5)

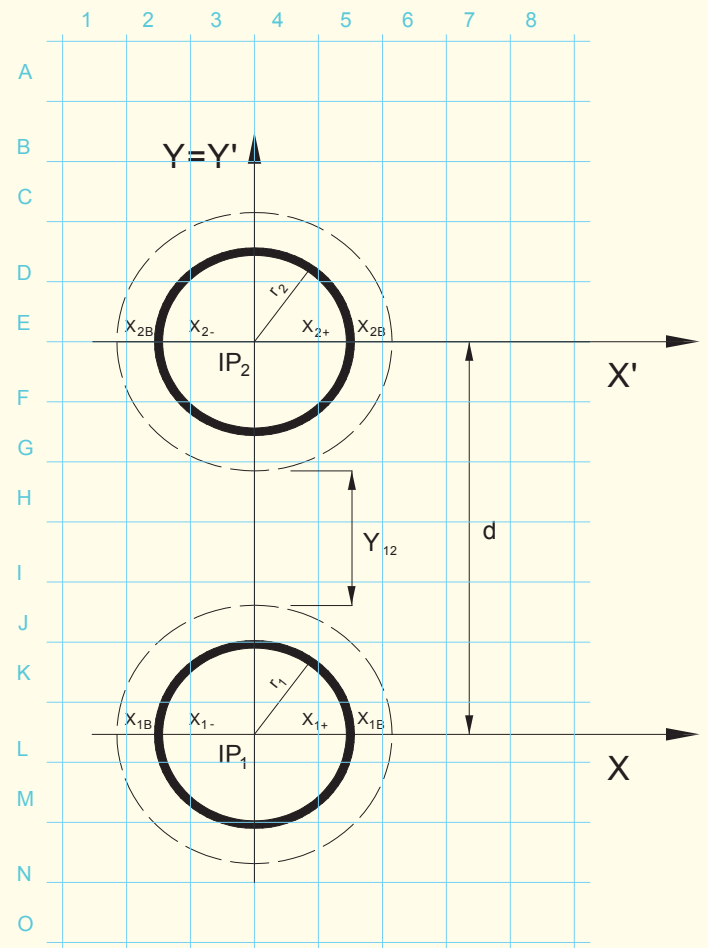

Fig. 5: Model of topological relations 2- $\mathrm{O}_{y}$

$$
\begin{aligned}
& I P_{1}=\left[x_{1}, y_{1}, 0\right]=[0,0,0] \quad I P_{2}=\left[x_{2}, y_{2}, 0\right] \\
& \alpha=\frac{\pi}{2} \\
& x_{2}=x_{1}=0 \\
& y_{2}=r_{1}+y_{1 B}+y_{2 B}+r_{2}+y_{12} \\
& d=r_{1}+y_{1 B}+y_{2 B}+r_{2}+y_{12} \\
& y_{12}=d-\left(r_{1}+y_{1 B}+y_{2 B}+r_{2}\right)
\end{aligned}
$$

$\mathrm{y}_{12}>0$ - this condition must be done because of the collision of constructed equipments of assembled system.
The charging point of constructed equipment of assembled system turned about angle $\alpha, \alpha$-angle of join charging points and axe $o_{x}:$ (Fig. 6)

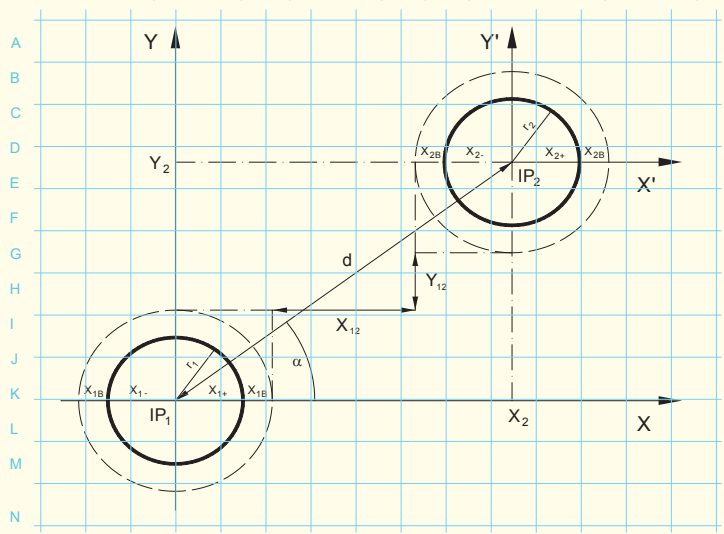

Fig. 6: Model of topological relations $2-\alpha$

$$
\begin{aligned}
& I P_{1}=\left[x_{1}, y_{1}, 0\right]=[0,0,0] \quad I P_{2}=\left[x_{2}, y_{2}, 0\right] \\
& \alpha \in\left(0, \frac{\pi}{2}\right) \\
& x_{2}=r_{1}+x_{1 B}+x_{2 B}+r_{2}+x_{12} \\
& y_{2}=r_{1}+y_{1 B}+y_{2 B}+r_{2}+y_{12} \\
& d=\sqrt{x_{2}^{2}+y_{2}^{2}} \\
& =\left(\left(r_{1}+x_{1 B}+x_{12}+x_{2 B}+r_{2}\right)^{2}\right. \\
& \left.\quad+\left(r_{1}+y_{1 B}+y_{12}+y_{2 B}+r_{2}\right)^{2}\right)^{\frac{1}{2}} \\
& x_{12}=x_{2}-\left(r_{1}+x_{1 B}+x_{2 B}+r_{2}\right) \\
& y_{12}=y_{2}-\left(r_{1}+y_{1 B}+y_{2 B}+r_{2}\right)
\end{aligned}
$$

$\mathrm{d}>\mathrm{r}_{1}+\mathrm{x}_{1 \mathrm{~B}}+\mathrm{r}_{2}+\mathrm{x}_{2 \mathrm{~B}}$ - this condition must be done because of the collision of constructed equipments of assembled system.

The distance between $\mathrm{x}_{12}$ and $\mathrm{y}_{12}$ are called reserve distances, about which we can change distance between charging points $\mathrm{IP}_{1}$ and $\mathrm{IP}_{2}$. The correction of location of second point will be by the modification of coordinate $\mathrm{x}_{2}$ about corrected distance $\mathrm{x}_{\mathrm{R}}$ (or by the modification of coordinate $\mathrm{y}_{2}$ about corrected distance $\mathrm{y}_{\mathrm{R}}$ ), but conditions must be done because of the collision of constructed equipments of assembled system. The corrected distance is number $x_{R}$ or $y_{R}$ from interval $\mathrm{x}_{\mathrm{R}} \in\left(0, \mathrm{x}_{12}\right)$ and $\mathrm{y}_{\mathrm{R}} \in\left(0, \mathrm{y}_{12}\right)$. The minimalization of distances and performance of safety distance between charging points is represented by the correlation of the location. The charging point $\mathrm{IP}_{2}$ is the beginning of 
coordinate system $\mathrm{IP}_{2}=\left[\mathrm{x}_{2}, \mathrm{y}_{2}, 0\right]=\mathrm{O}_{\mathrm{xyz}}$ its coordinates are $[0,0,0]$ in the new coordinate system. The progress of the charge another point is analogical.

\section{The Model of Topological Relations Applied to Five Constructed Equipment of Assembled System}

The modeling of topological relations of five constructed equipment of assembled systems (cellular organized model) go out of these assumptions: (Fig. 7) - The firs step is to select the first localized constructed equipment (robot or operating staff). The charging point of constructed equipment is the beginning of coordinate system $I P=O_{x y z}$.

- The orientation of coordinate system of creating structure is identical with the orientation of coordinate system of embedded constructed equipment. Is given: $x=x_{R}$ resp. $x=x_{C L}, y=y_{R}, y=y_{C L}$, whereas $x_{R}, y_{R}$ - vectors $x$-axe and $y$-axe of coordinate system of constructed equipment (e.g. robot at automatized assembly systems) $x_{C L}, y_{C L}$-vectors $x$-axe and $y$-axe of coordinate system of constructed equipment (e.g. human at assembly system supported by human assist).

- There is selected from the database the second constructed equipment and setting the coordinates of its charging point (considering the first constructed equipment), in which is objective equipment localizated $I P_{2}=\left[x_{2}, y_{2}, z_{2}\right]$.

- The charging point of the second constructed equip- ment is the beginning of new coordinate system.

- The selection of next constructed equipment from the database.

Analogously, there is setting the coordinates of charging point, in which is the next constructed equipment placed.

Values of dynamic parameters, i.e. parameters variable by time, if is needed, are setting from equations of motion.

General linear motion:

$x=x_{0}+v t, v=v_{0}, a=0$

Accelerated linear motion:

$x=x_{0}+\frac{1}{2} a t^{2}, v=a t, a=a_{0}$

Combination of general linear motion and accelerated linear motion:

$x=x_{0}+v_{0} t+\frac{1}{2} a t^{2}, v=v_{0}+a t, a=a_{0}$

Rotary movement:

$v=\omega r, \varphi=\omega t$

The utilization of these equations facilitates the simulation of mobile parts position of assembled units or manipulation equipments in selected time moment $\mathrm{t}$

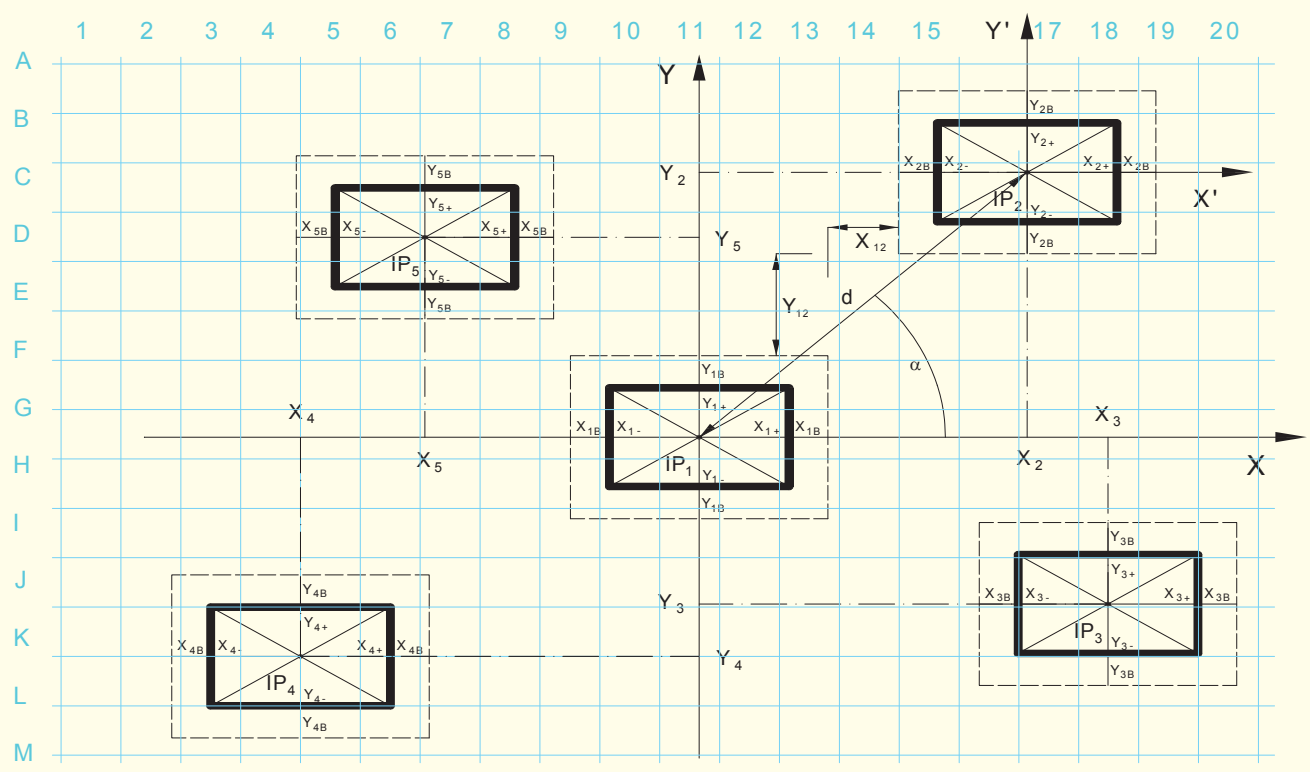

Fig. 7: Model of the topological relations for 5 objects 
by known beginning conditions and by defined motion type.

If the first charging point is placed to the beginning of coordinate system, than there are four possibilities regarding to plain partition to place the second point. The charging point is possible to place to:

\begin{tabular}{|c|c|c|c|}
\hline I. quadr. & $\alpha \in\left(0, \frac{\pi}{2}\right)$ & II. quadr. & $\alpha \in\left(\frac{\pi}{2}, \pi\right)$ \\
\hline III. quadr. & $\alpha \in\left(\pi, \frac{3 \pi}{2}\right)$ & IV. quadr. & $\alpha \in\left(\frac{3 \pi}{2}, 2 \pi\right)$ \\
\hline
\end{tabular}

I. Quadrant (if the charging point is placed to right up direction from cardinal equipment):

$$
\begin{aligned}
& I P_{2}=\left[x_{2}, y_{2}, 0\right] \\
& x_{2}=x_{1+}+x_{1 B}+x_{2 B}+x_{2-}+x_{12} \\
& y_{2}=y_{1+}+y_{1 B}+y_{2 B}+y_{2-}+y_{12}
\end{aligned}
$$

II. Quadrant (if the charging point is placed to left up direction from the cardinal equipment):

$I P_{5}=\left[x_{5}, y_{5}, 0\right]$

$x_{5}=-\left(x_{1-}+x_{1 B}+x_{5 B}+x_{5+}+x_{15}\right)$

$y_{5}=y_{1+}+y_{1 B}+y_{5 B}+y_{5-}+y_{15}$

III. Quadrant (if the charging point is placed to left down from cardinal equipment):

$$
\begin{aligned}
& I P_{4}=\left[x_{4}, y_{4}, 0\right] \\
& x_{4}=-\left(x_{1-}+x_{1 B}+x_{4 B}+x_{4+}+x_{14}\right) \\
& y_{4}=-\left(y_{1-}+y_{1 B}+y_{4 B}+y_{4+}+y_{14}\right)
\end{aligned}
$$

IV. Quadrant (if the charging point is placed to right down from cardinal equipment):

$$
\begin{aligned}
& I P_{3}=\left[x_{3}, y_{3}, 0\right] \\
& x_{3}=-\left(x_{1-}+x_{1 B}+x_{3 B}+x_{3+}+x_{13}\right) \\
& y_{3}=-\left(y_{1-}+y_{1 B}+y_{3 B}+y_{3+}+y_{13}\right)
\end{aligned}
$$

The distance for two charging points is given: (i.e. for $I P_{1}$ and $\left.I P_{2}\right)$

$$
\begin{aligned}
d & =\sqrt{x_{2}^{2}+y_{2}^{2}} \\
& =\left(\left(x_{1+}+x_{1 B}+x_{12}+x_{2 B}+x_{2-}\right)^{2}\right. \\
& \left.+\left(y_{1+}+y_{1 B}+y_{12}+y_{2 B}+y_{2-}\right)^{2}\right)^{\frac{1}{2}}
\end{aligned}
$$

$$
\begin{aligned}
& d=\frac{y_{1+}+y_{1 B}+y_{12}+y_{2 B}+y_{2-}}{\sin \alpha} \\
& d=\frac{x_{1+}+x_{1 B}+x_{12}+x_{2 B}+x_{2-}}{\cos \alpha}
\end{aligned}
$$

By placing into particular quadrant must be done the inequations for $\mathrm{x}$-axe and $\mathrm{y}$-axe coordinates to observance of safety distances.

$$
\begin{aligned}
& x_{2}-x_{1}>x_{1+}+x_{B 1}+x_{B 2}+x_{2-} \\
& x_{3}-x_{1}>x_{1+}+x_{B 1}+x_{B 3}+x_{3-} \\
& \left|x_{4}-x_{1}\right|>x_{1-}+x_{B 1}+x_{B 4}+x_{4+} \\
& \left|x_{5}-x_{1}\right|>x_{1-}+x_{B 1}+x_{B 5}+x_{5+} \\
& y_{2}-y_{1}>y_{1+}+y_{B 1}+y_{B 2}+y_{2-} \\
& \left|y_{3}-y_{1}\right|>y_{1+}+y_{B 1}+y_{B 3}+y_{3+} \\
& \left|y_{4}-y_{1}\right|>y_{1-}+y_{B 1}+y_{B 4}+y_{4+} \\
& y_{5}-y_{1}>y_{1+}+y_{B 1}+y_{B 5}+y_{5-}
\end{aligned}
$$

\section{Modeling of Complex Structures of Assembly Systems}

The proposed models are reference methods, which is utilized for solution of topological relations between constructed equipment for more complex structures of assembly systems.

\subsection{Modeling of opened in-line structures of assembly sys-} tems

The modeling of topological relations of assembled equipment with opened in-line structures go out these assumptions:

- The beginning of the coordinate system $O_{x y z}$ of assembly line is in the position of elementary constructed equipment. The elementary constructed equipment is that one, which shows the minimal $x$-axe and $y$-axe coordinates and is placed into referential space as the first.

- The orientation of coordinate system of assembly line is identical with the orientation of coordinate system of elementary constructed equipment.

- The charging point of line is identical with the charging point of elementary constructed equipment.

- The first constructed equipment is placed into working zone as the $I P_{1}=\left[x_{1}, y_{1}, z_{1}\right]=[0,0,0]$ point.

- The second equipment is placed into point $I P_{2}=\left[x_{2}, y_{2}, z_{2}\right]$. The coordinates $x_{2}, y_{2}$ are defined analogously to previous cases:

For equipment placed into $\mathrm{x}$-axe is given: 
$x_{2}=x_{1+}+x_{i B}+x_{2 B}+x_{2-}+x_{12}$

$y_{2}=y_{1}=0$

For equipment placed into $\mathrm{y}$-axe is given:

$x_{2}=x_{1}=0$

$y_{2}=y_{1+}+y_{i B}+y_{2 B}+y_{2-}+y_{12}$

The second constructed equipment - its charging point or marginal point is identical with the beginning of new coordinate system.

Analogously, the third point is placed as the second one with respect of the first constructed equipment.

The coordinates of the beginning of new coordinate system with respect of aboriginal coordinate system can be calculated by elementary transformation relations for translation, rotation etc..

- For $i$ equipment at chosen axe is given: $\mathrm{x}$-axe $\mathrm{O}_{\mathrm{x}}$ :

$x_{i}=x_{i-1}+x_{(i-1)}++x_{(i-1) B}+x_{i, i-1}+x_{i B}+x_{i-}$

$y_{i}=y_{i-1}$

$\mathrm{y}$-axe $\mathrm{o}_{\mathrm{y}}$ :

$x_{i}=x_{i-1}$

$y_{i}=y_{i-1}+y_{(i-1)}++y_{(i-1) B}+y_{i, i-1}+y_{i B}+y_{i-}$

By following this procedure of placing constructed equipment into particular axe can be built up the inline structure consisting of $i$ constructed equipment.

\subsection{Semi-opened in-line structure (U - structure)}

The modeling of topological relations of assembled equipment with $U$ structures go out these assumptions:

The beginning of the coordinate system $O_{x y z}$ of assembly $U$-line is in the position of elementary constructed equipment. The elementary constructed equipment is that one, which shows the minimal $x$-axe and $y$-axe coordinates and is placed into referential space as the first.

The orientation of coordinate system of assembly line is identical with the orientation of coordinate system of elementary constructed equipment.

The charging point of line is identical with the charging point of elementary constructed equipment.

The first constructed equipment is placed into working zone as the $I P_{1}=\left[x_{1}, y_{1}, z_{1}\right]=[0,0,0]$ point.

The second equipment is placed into point
$I P_{2}=\left[x_{2}, y_{2}, z_{2}\right]$. The coordinates $x_{2}, y_{2}$ are defined analogously to previous cases:

$$
\begin{aligned}
& x_{2}=x_{1+}+x_{i B}+x_{2 B}+x_{2-}+x_{12} \\
& y_{2}=y_{1+}+y_{i B}+y_{2 B}+y_{2-}+y_{12}
\end{aligned}
$$

- The third equipment is placed into $I P_{3}=\left[x_{3}, y_{3}, 0\right]$. For coordinates $x_{3}$ and $y_{3}$ is given: $x_{3}=x_{2}, y_{3}=-y_{2}$. For $i$ equipment is given:

$$
\text { If } \mathrm{i}=2 \mathrm{k} \text { : }
$$

$x_{i}=x_{i-2}+x_{(i-2)^{+}}+x_{(i-2) B}+x_{i, i-2}+x_{i B}+x_{i-}$

$y_{i}=y_{i-2}$

$$
\text { If } \mathrm{i}=2 \mathrm{k}+1 \text { : }
$$

$x_{i}=x_{i-1}$

$y_{i}=-y_{i-1}$

By following this procedure of placing constructed equipment into particular axe can be built up the $U$ line structure consisting of $i$ constructed equipment ( $i$ - odd number).

\subsection{Closed - line structure (ring structure)}

The modeling of topological relations of assembled equipment with ring structures go out these assumptions:

- The beginning of the coordinate system $O_{x y z}$ of assembly line is in the position of elementary constructed equipment. The elementary constructed equipment is that one, which shows the minimal $x$-axe and $y$-axe coordinates and is placed into referential space as the first.

- The orientation of coordinate system of assembly line is identical with the orientation of coordinate system of elementary constructed equipment.

- The charging point of line is identical with the charging point of elementary constructed equipment.

The first constructed equipment is placed into working zone as the $I P_{i}=\left[x_{i}, y_{i}, z_{i}\right]=[0,0,0]$ point.

$$
\begin{aligned}
& \mathrm{IP}_{n}=\left[x_{n}, y_{n}, 0\right] \\
& y_{n}=0 \\
& x_{n}=x_{\max }
\end{aligned}
$$

- The constructed equipments are placed as in the case of $U$-line and the last placed equipment is with the 
maximum $x$-axe coordinate of charging point.

- The constructed equipments can be placed into ring - structure by adequate organization of two $U$-lines.

\subsection{Cell structure}

The modeling of topological relations of assembled equipment with cell structures go out these assumptions:

- The beginning of the coordinate system $O_{x y z}$ of assembly cell structure is in the position of basic constructed equipment. The basic constructed equipment is that one, which shows the minimal $x$-axe and $y$-axe coordinates and is placed into referential space as the first.

- The orientation of coordinate system of assembly line is identical with the orientation of coordinate system of basic constructed equipment.

- The charging point of line is identical with the charging point of basic constructed equipment.

The first constructed equipment is placed into working zone as the $I P_{i}=\left[x_{i}, y_{i}, z_{i}\right]=[0,0,0]$ point.

For equipment placed to the right up direction from basic equipment $I P_{i}=\left[x_{i}, y_{i}, 0\right]$ is given:

$\mathrm{IP}_{j}=\left[x_{j}, y_{j}, 0\right]$

$x_{j}=x_{i+}+x_{i B}+x_{j B}+x_{j-}+x_{i j}$

$y_{j}=y_{i+}+y_{i B}+y_{j B}+y_{j-}+y_{i j}$

- For equipment placed to the left up direction from basic equipment $I P_{i}=\left[x_{i}, y_{i}, 0\right]$ is given:

$\mathrm{IP}_{j}=\left[x_{j}, y_{j}, 0\right]$

$x_{j}=-\left(x_{i-}+x_{i B}+x_{j B}+x_{j+}+x_{i j}\right)$

$y_{j}=y_{i+}+y_{i B}+y_{j B}+y_{j-}+y_{i j}$

- For equipment placed to the left down direction from basic equipment $I P_{i}=\left[x_{i}, y_{i}, 0\right]$ is given:

$\mathrm{IP}_{j}=\left[x_{j}, y_{j}, 0\right]$

$x_{j}=-\left(x_{i-}+x_{i B}+x_{j B}+x_{j+}+x_{i j}\right)$

$y_{j}=-\left(y_{i-}+y_{i B}+y_{j B}+y_{j+}+y_{i j}\right)$

For equipment placed to the right down direction from basic equipment $I P_{i}=\left[x_{i}, y_{i}, 0\right]$ is given:

$\mathrm{IP}_{j}=\left[x_{j}, y_{j}, 0\right]$

$x_{j}=x_{i+}+x_{i B}+x_{j B}+x_{j-}+x_{i j}$

$y_{j}=-\left(y_{i-}+y_{i B}+y_{j B}+y_{j+}+y_{i j}\right)$
For the distance between two charging points $I P_{i}$ and $I P_{j}$ is given:

$$
\begin{gathered}
d=\left(\left(x_{\mathrm{i}+}+x_{i B}+x_{i j}+x_{j B}+x_{\mathrm{j}-}\right)^{2}\right. \\
\left.+\left(y_{\mathrm{i}+}+y_{i B}+y_{i j}+y_{j B}+y_{\mathrm{j}-}\right)^{2}\right)^{\frac{1}{2}} \\
d=\frac{y_{i+}+y_{i B}+y_{i j}+y_{j B}+y_{j-}}{\sin \alpha}
\end{gathered}
$$

or

$d=\frac{x_{i+}+x_{i B}+x_{i j}+x_{j B}+x_{j-}}{\cos \alpha}$

- For angle size $\alpha$ (turning angle) is given:

$$
\begin{aligned}
& \operatorname{tg} \alpha=\frac{y_{i+}+y_{i B}+y_{i j}+y_{j B}+y_{j-}}{x_{i+}+x_{i B}+x_{i j}+x_{j B}+x_{j-}} \\
& \Rightarrow \alpha=\operatorname{arctg} \frac{y_{i+}+y_{i B}+y_{i j}+y_{j B}+y_{j-}}{x_{i+}+x_{i B}+x_{i j}+x_{j B}+x_{j-}}
\end{aligned}
$$

If the distance $d$ between two charging points and angle $\alpha$ is known, for the coordinates of charging point $I P_{j}=\left[x_{j}, y_{j}, 0\right]$ is given:

$x_{j}=d \cos \alpha$

$y_{j}=d \sin \alpha$

and $\mathrm{x}$-axe and $\mathrm{y}$-axe distances:

$$
\begin{aligned}
& x_{i j}=x_{j}-x_{i_{+}}-x_{i B}-x_{j B}-x_{j-} \\
& y_{i j}=y_{j}-y_{i+}-y_{i B}-y_{j B}-y_{j-}
\end{aligned}
$$

The example of assembled system created on the base of optimalization of topological relations of constructed equipments is displayed in Fig. 8. This threedimensional model was created in program AUTOCAD.

\section{Conclusion}

The suggested models of topological relations of constructed equipments of assembled models provide possibility of optimalization of location problems. The models of topological relations in assembled systems are characterized of universality in application conditions and are suitable for designing automated assembled systems. 


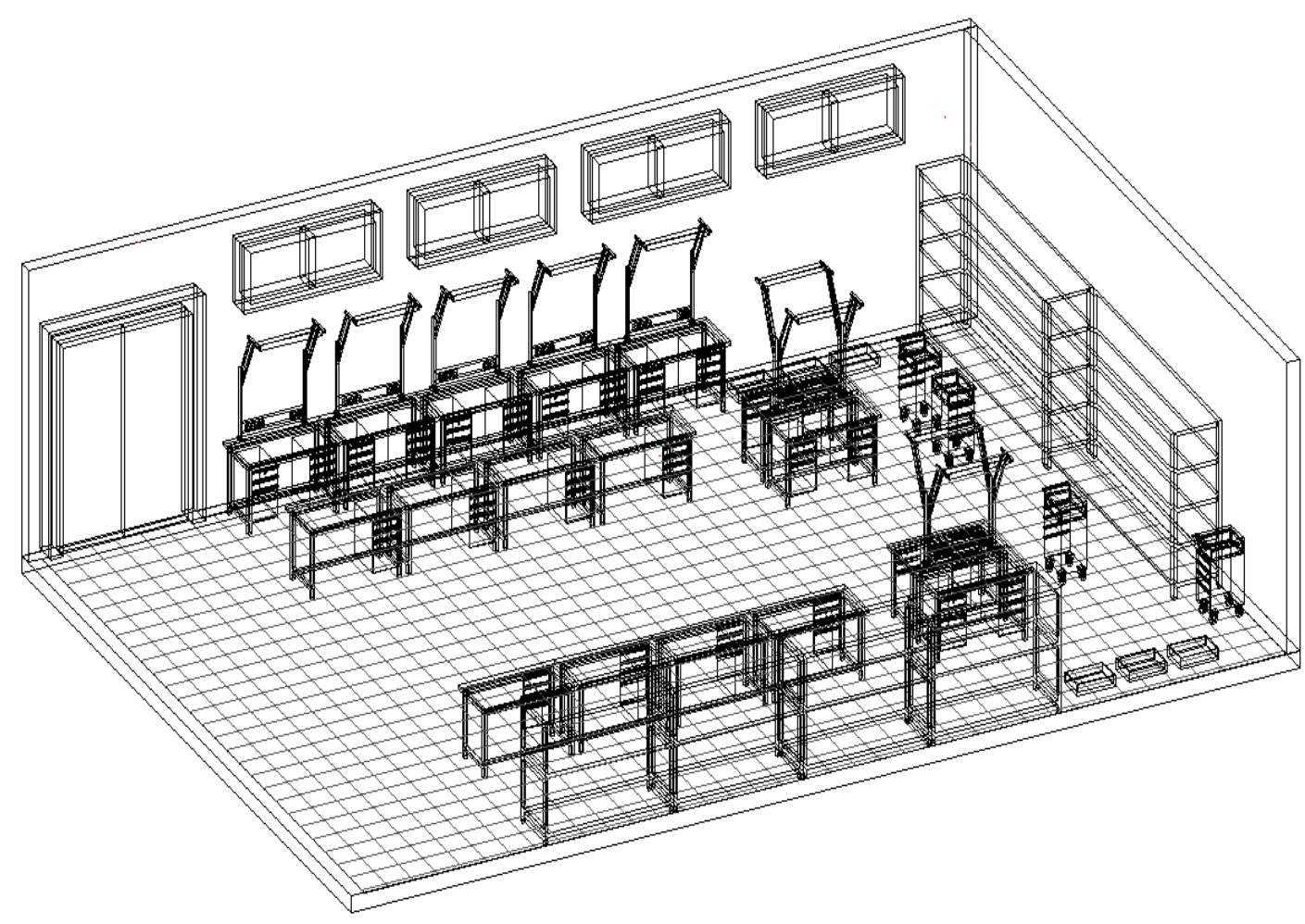

Fig. 7: The example of the reference area of assembled system based of the utilizing model of topological relations of constructed equipments

\section{References}

Filo, M., Lukáč, M.: Modeling and Simulation of Mechanism with Computer Aid. University of Zilina, 2005.

Kováč, J., Svoboda, M., Liška, O.: Automated and Flexibile Assembly. Vienala Kosice, 2000.

Kuric, I., Košturiak, J., Janáč, A., Peterka, J., Marcinčin, J.: Computer Aided Systems Applied in Machinery Engineering. Edis Zilina, 2002. 


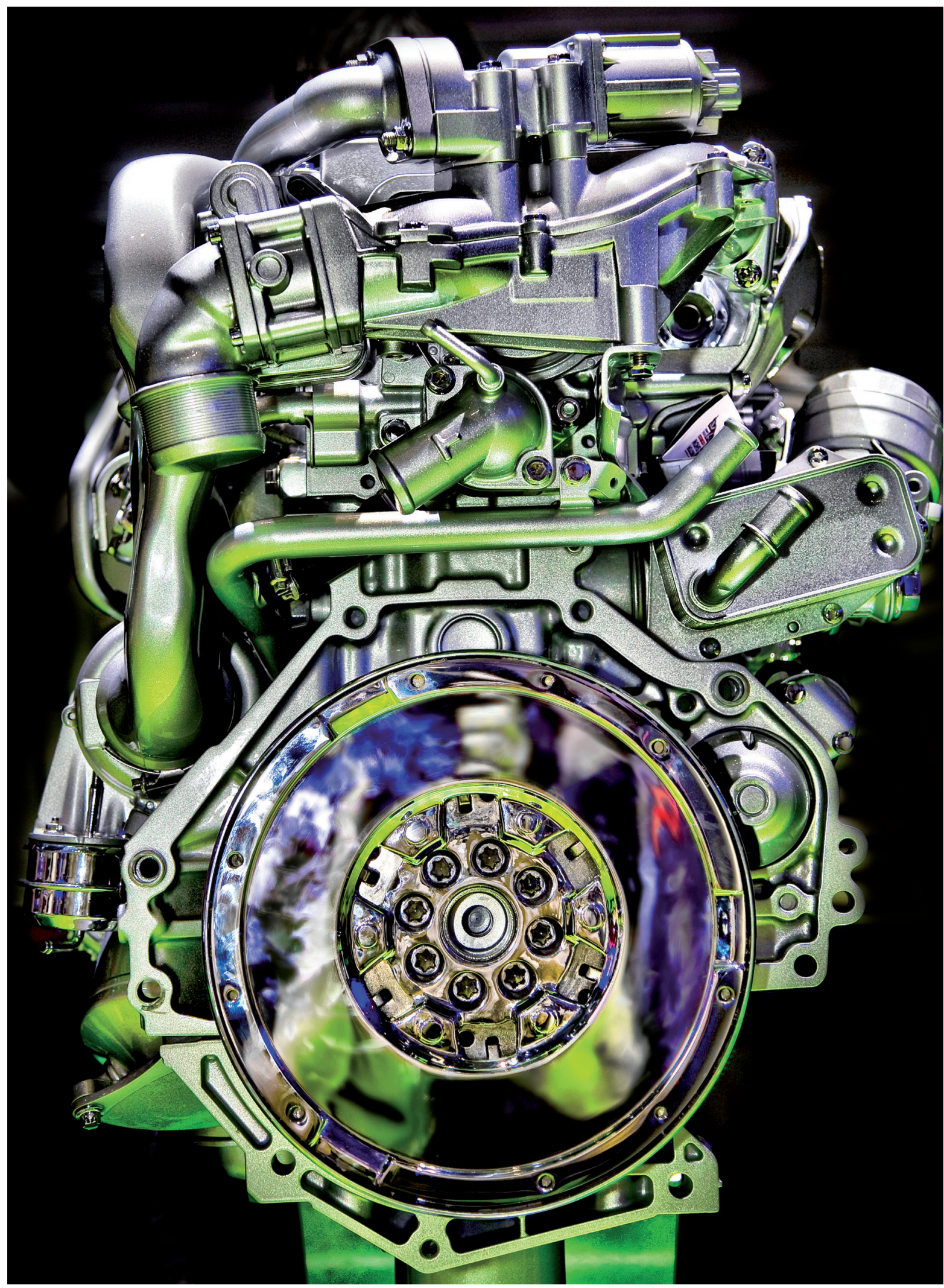

